\title{
Relação entre solos afetados por sais e concentração de metais pesados em quatro perímetros irrigados no Ceará ${ }^{1}$
}

\author{
Cleyton S. M. Cunha ${ }^{2}$, Fernando F. F. Hernadez ${ }^{3}$, Francisco N. da Silva ${ }^{4}$, \\ Maria E. O. Escobar ${ }^{5}$, Diego R. Magalhães ${ }^{6} \&$ David C. dos Anjos \\ ${ }^{1}$ Trabalho selecionado do II INOVAGRI International Meeting, realizado de 13 a 16 de abril de 2014, Fortaleza - CE, Brasil \\ ${ }^{2}$ UFC. Fortaleza, CE. E-mail: cleytonsaialy@ig.com.br \\ ${ }^{3}$ UFC. Fortaleza, CE. E-mail: ferrey@ufc.br \\ ${ }^{4}$ UNILAB. Redenção, CE. E-mail: nildo@unilab.edu.br (Autor correspondente) \\ ${ }^{5}$ UFC. Fortaleza, CE. E-mail: mariaeugenia@ufc.br \\ ${ }^{6}$ UFC. Fortaleza, CE. E-mail: diego_rolney@hotmail.com \\ ${ }^{7}$ UFC. Fortaleza, CE. E-mail: david_agronomia@hotmail.com
}

\section{Palavras-chave:}

metais pesados

salinização

análise multivariada

\section{R E S U M O}

O trabalho teve como objetivo verificar a relação entre atributos químicos relacionados com a salinidade (CTC, pH, PST e CE) e concentrações de metais pesados ( $\mathrm{Fe}, \mathrm{Al}, \mathrm{Si}, \mathrm{Ti}, \mathrm{Mn}, \mathrm{Zn}, \mathrm{Cr}, \mathrm{V}, \mathrm{Ni}, \mathrm{Cu}, \mathrm{Co}, \mathrm{B}, \mathrm{Pb}, \mathrm{Se}, \mathrm{Cd}, \mathrm{Mo}$, $\mathrm{Zr}$ ) e suas inter-relações em solos afetados por sais relativo a quatro perímetros irrigados no Ceará. Foram coletadas amostras de solo, de diferentes texturas, nas profundidades de 0-10 e 10-20 cm em 10 pontos dentro de cada perímetro, totalizando oitenta (80) amostras. Foram utilizadas a análise de correlação canônica (ACC) e a análise fatorial com extração dos fatores em componentes principais (AF) para verificar as possíveis relações entre os dois conjuntos de variáveis. Os atributos do solo relacionadas com a salinidade contribuíram para explicar as concentrações de metais pesados nos solos dos quatro perímetros irrigados, quando analisados em conjunto; todavia, não foram capazes de explicar quando analisados de forma individual; seguindo a ordem de importância: $\mathrm{CTC}>\mathrm{pH}>\mathrm{PST}>\mathrm{CE}$. Foram verificadas correlações entre os elementos metálicos analisados neste estudo (B, Mo, Si, Zr, Cr, Ni, Pb, Ti, Zn, Cd, Co, Se e Cu) com o Fe, Mn, Al, Ti e V, ou, ainda, afinidades geoquímicas entre os elementos.

\section{Key words:}

heavy metals

salinization

multivariate analysis

\section{Relationship between salt affected soils and concentration of heavy metals in four irrigated districts of Ceará}

\begin{abstract}
A B S T R A C T
The study aimed to investigate the relationship between chemical attributes related to salinity $(\mathrm{CEC}, \mathrm{pH}, \mathrm{EC}$ and ESP) and concentrations of heavy metals (Fe, $\mathrm{Al}, \mathrm{Si}, \mathrm{Ti}, \mathrm{Mn}, \mathrm{Zn}, \mathrm{Cr}, \mathrm{V}, \mathrm{Ni}, \mathrm{Cu}, \mathrm{co}, \mathrm{B}, \mathrm{Pb}, \mathrm{Se}, \mathrm{Cd}, \mathrm{Mo}, \mathrm{Zr}$ ) and their inter-relationships in salt affected soils relative to four (4) irrigated areas in Ceará State. Soil samples of different textures from 0-10 and 10-20 cm were collected at 10 points within each district, totaling eighty $(80)$ samples. The canonical correlation analysis (CCA) and factorial analysis with factor extraction on principal components (AF) to determine possible relationships between the two sets of variables were used. Soil attributes related to salinity contributed to explain the concentrations of heavy metals in soils of the studied areas when analysed all together, but not when analysed individually, following the order of importance: $\mathrm{CEC}>\mathrm{pH}>\mathrm{ESP}>\mathrm{EC}$. Correlations were found between the analysed metals in this study (B, Mo, Si, $\mathrm{Zr}, \mathrm{Cr}, \mathrm{Ni}, \mathrm{Pb}, \mathrm{Ti}, \mathrm{Zn}, \mathrm{Cd}, \mathrm{Co}$, Se and $\mathrm{Cu}$ ) with $\mathrm{Fe}, \mathrm{Mn}$, $\mathrm{Al}, \mathrm{Ti}$ and $\mathrm{V}$, or furthermore, geochemical affinities between the elements.
\end{abstract}

\section{INTRODUÇÃO}

O processo de acumulação de sais no solo é um problema relevante no cenário mundial e vem acarretando em perdas significativas na agricultura em produção e produtividade, principalmente em áreas áridas e semiáridas onde os sais solúveis se precipitam na superfície ou no interior do solo. Nos perímetros irrigados é frequente o surgimento de solos afetados por sais devido ao manejo ineficiente do solo e da água, a drenagem deficiente em decorrência da baixa condutividade hidráulica dos solos, as condições topográficas desfavoráveis e a constante exploração agrícola das terras. Desta forma, muitos perímetros irrigados estão propensos à inundação e a elevação do lençol freático (Leite et al., 2007).

Com o aumento da salinidade do solo pode aumentar a mobilização de metais e promover a absorção de metais pelas

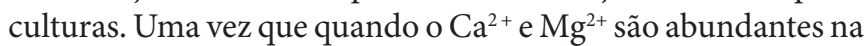
solução do solo, assim os metais podem ser mobilizados como resultado da concorrência entre estes cátions divalentes e os íons metálicos (Paalman et al., 1994). 
Com o intuito de elucidar esse problema, propõe-se o uso de técnicas de análise estatística multivariada, como análise de correlação canônica e análise fatorial, para demonstrar como grupos de variáveis interagem e como interagem entre si, levando-se em conta a contribuição conjunta de atributos químicos e físicos associados à migração e a adsorção de metais pesados nos solos. As técnicas de análise multivariadas já foram utilizadas em estudos geoquímicos (Fadigas et al., 2002) e de inter-relações entre atributos de solo, verificando inferências sobre conjuntos de dados em nível de significância conhecido, permitindo assim ampla faixa de compreensão da distribuição geral dos resultados (Yongming et al., 2005).

O presente estudo buscou estabelecer relações entre solos afetados por sais, pelas variáveis (CTC, PST, pH e CE) e concentração de metais pesados (Al, B, Hg, Mn, Mo, Si, Zr, $\mathrm{Cr}, \mathrm{Fe}, \mathrm{V}, \mathrm{Ni}, \mathrm{Pb}, \mathrm{Ti}, \mathrm{Zn}$, As, Se, Cd, Co e $\mathrm{Cu}$ ) em amostras de Neossolos Flúvicos e Quartizarênicos referentes a quatro perímetros irrigados no Ceará, a partir de técnicas de análise multivariada.

\section{Material e Métodos}

Levando-se em consideração os lotes irrigados e a uniformidade do terreno, foi definido o contorno para amostragem dos solos. Entre os meses de dezembro de 2012 a fevereiro de 2013, foram feitas visitas nas áreas dos Perímetros Irrigados Morada Nova, Baixo-Acaraú, Curu-Pentecoste e IcóLima Campos, respectivamente. Os serviços de administração, operação e manutenção da infra-estrutura de uso dos mesmos foram iniciados entre os anos de 1970 a 2001 (ADECE, 2011).

A coleta realizou-se seguindo o curso dos rios, onde os solos dos lotes apresentavam visivelmente maiores teores de sais dentro dos perímetros irrigados. Foram coletadas amostras de solo nas profundidades de 0-10 e 10-20 cm em 10 pontos em cada perímetro (totalizando 80 amostras), com auxílio de trado holandês de aço inox. Tais pontos foram bem espaçados, em lotes diferentes, de forma que obtivesse uma representatividade dos solos dos perímetros.

As amostras, após secas ao ar, foram passadas em peneiras de nylon com abertura de $2 \mathrm{~mm}$. Alíquota deste material foi macerada em almofariz de ágata e passado em peneiras de $0,3 \mathrm{~mm}$ de abertura (ABNT ${ }^{\circ}$ 50), com malha de aço inoxidável, visando evitar contaminações. Foram coletados aproximadamente $300 \mathrm{~kg}$ de solo, sendo $150 \mathrm{~kg}$ na camada de $0-10$ e $150 \mathrm{~kg}$ na camada de $10-20 \mathrm{~cm}$ em cada perímetro selecionado.

A digestão das amostras do estudo e da amostra certificada, (NIST SRM 2709 San Joaquin soil) para a obtenção dos teores de metais pesados, baseou-se no método 3050 B (USEPA, 1998) (Tabelas 1 e 2). Para este procedimento, utilizou-se $0,5 \mathrm{~g}$ da amostra digerida em $5 \mathrm{~mL}$ de $\mathrm{HNO}_{3}$ concentrado em tubos de digestão em bloco digestor aberto por $10 \mathrm{~min}$ a $95 \pm 5^{\circ} \mathrm{C}$, então as amostras foram resfriadas por 15 min e novamente adicionados $5 \mathrm{~mL}$ de $\mathrm{HNO}_{3}$. Estas foram conduzidas ao bloco digestor para aquecimento a $95 \pm 5{ }^{\circ} \mathrm{C}$ por mais $2 \mathrm{~h}$. Após este período as amostras foram resfriadas por 30 min e então
Tabela 1. Faixa de variação (FV) dos teores de metais pesados referente às oitenta amostras de solo dos quatro perímetros irrigados do Ceará

\begin{tabular}{|c|c|c|c|}
\hline Elementos & $\mathrm{FV}\left(\mathrm{mg} \mathrm{kg}^{-1}\right)$ & Elementos & $\mathrm{FV}\left(\mathrm{mg} \mathrm{kg}^{-1}\right)$ \\
\hline $\mathrm{Cu}$ & $0-45,58$ & $\mathrm{Si}$ & $145,32-1293,51$ \\
\hline $\mathrm{Ni}$ & $0-51,52$ & V & $0,01-79,74$ \\
\hline Co & $0,11-40,29$ & B & $0,14-53,96$ \\
\hline $\mathrm{Fe}$ & $103,74-20946,33$ & Mo & $0,01-1,05$ \\
\hline $\mathrm{Zn}$ & $2,43-150,96$ & $\mathrm{Ti}$ & $6,68-893,14$ \\
\hline $\mathrm{Mn}$ & $0,25-1211,68$ & $\mathrm{Se}$ & $0,01-4,47$ \\
\hline $\mathrm{Cr}$ & $0,99-77,98$ & $\mathrm{Zr}$ & $0,01-0,41$ \\
\hline $\mathrm{Pb}$ & $0,08-2,97$ & $\mathrm{Hg}$ & - \\
\hline $\mathrm{Cd}$ & $0,01-1,13$ & As & - \\
\hline $\mathrm{Al}$ & $791,97-24791,83$ & & \\
\hline
\end{tabular}

- : Não foi detectado pelo aparelho

Tabela 2. Faixa de concentração e teor médio de metais pesados certificados (NIST SRM 2709 San Joaquin soil) pela US Departament of Commerce utilizados nas análises pelo método USEPA (3050 B)

\begin{tabular}{|c|c|c|c|c|}
\hline \multirow{2}{*}{ Elementos } & FVVC (NIST) & VCM (NIST) & TTM & \multirow{2}{*}{$\begin{array}{c}\text { VRM }^{1} \\
\text { (\%) }\end{array}$} \\
\hline & & $\mathrm{mg} \mathrm{kg}^{-1}$ & & \\
\hline Al & $13.000-17.000$ & 15.000 & $13.369,56$ & 80 \\
\hline B & $\star$ & * & 29,14 & * \\
\hline $\mathrm{Hg}$ & $0,79-0,92$ & 0,85 & 0,84 & 99 \\
\hline $\mathrm{Mn}$ & $380-450$ & 415 & 420,29 & 101 \\
\hline Mo & * & * & 1,06 & * \\
\hline $\mathrm{Si}$ & * & * & 318,92 & * \\
\hline $\mathrm{Zr}$ & * & * & 0,51 & $\star$ \\
\hline $\mathrm{Cr}$ & $46-67$ & 56.5 & 68,50 & 121 \\
\hline $\mathrm{Fe}$ & $22.000-2.6000$ & 24.000 & $17.329,30$ & 73 \\
\hline V & $43-71$ & 57 & 55,38 & 97 \\
\hline $\mathrm{Ni}$ & $59-71$ & 65 & 73,81 & 113 \\
\hline $\mathrm{Pb}$ & $8,1-10$ & 9,05 & 4,23 & 47 \\
\hline $\mathrm{Ti}$ & * & * & 42,52 & * \\
\hline $\mathrm{Zn}$ & $69-87$ & 78 & 86,64 & 111 \\
\hline As & $6,4-10$ & 8,2 & 7,52 & 91 \\
\hline Se & $0,69-1,9$ & 1,3 & 1,70 & 130 \\
\hline $\mathrm{Cd}$ & $0,33-0,66$ & 0,5 & 0,50 & 100 \\
\hline Co & $8,2-13$ & 10,6 & 12,93 & 121 \\
\hline $\mathrm{Cu}$ & $24-28$ & 26 & 20,09 & 78 \\
\hline
\end{tabular}

$1 \% \mathrm{R}=$ (valor recuperado médio/valor certificado médio) $\times 100$; *Valores não fornecidos pelo NIST (2002). FVVC - faixa de variação dos valores certificados; VCM - valor certificado médio; TTM - teores totais médios; VRM - valor recuperado médio.

adicionados $1 \mathrm{~mL}$ de água destilada e $1,5 \mathrm{~mL}$ de $\mathrm{H}_{2} \mathrm{O}_{2}$ a $30 \%$. Após cessar a efervescência, foi adicionado mais $5 \mathrm{~mL}$ de $\mathrm{H}_{2} \mathrm{O}_{2}$ e as amostras foram levadas novamente ao bloco digestor por mais $2 \mathrm{~h}$ a $95 \pm 5^{\circ} \mathrm{C}$. Por fim, as amostras foram resfriadas e receberam $10 \mathrm{~mL}$ de $\mathrm{HCl}$ concentrado e $20 \mathrm{~mL}$ de água destilada e foram aquecidas por $15 \mathrm{~min}$ a $95 \pm 5^{\circ} \mathrm{C}$. As alíquotas foram resfriadas, filtradas e armazenadas em recipiente plástico. Foi utilizado o ICP - ACAP 6300 DUO da Thermo Scientific para a determinação dos metais pesados e suas concentrações.

As análises de $\mathrm{pH}$ em água $(1: 2,5)$, cátions trocáveis $\left(\mathrm{Ca}^{2+}\right.$, $\mathrm{Mg}^{2+}, \mathrm{K}^{+}, \mathrm{Na}^{+}$), acidez potencial $\left(\mathrm{Al}^{3+}+\mathrm{H}^{+}\right)$, condutividade elétrica 1:1 (CE), percentagem de sódio trocável (PST), capacidade de troca de cátions (CTC), análise granulométrica e carbono orgânico (CO) foram realizadas segundo EMBRAPA (1997) (Tabela 3).

Os resultados foram avaliados por meio de análises de correlações canônicas, adotando-se dois grupos: O grupo um 
Tabela 3. Valores médios das características físico-químicas referentes às oitenta amostras do estudo

\begin{tabular}{|c|c|c|c|c|c|c|c|c|}
\hline & \multicolumn{2}{|c|}{ Morada Nova } & \multicolumn{2}{|c|}{ Baixo-Acaraú } & \multicolumn{2}{|c|}{ Curu-Pentecoste } & \multicolumn{2}{|c|}{ Icó-Lima-Campos } \\
\hline & \multicolumn{8}{|c|}{ Profundidade $(\mathrm{cm})$} \\
\hline & $0-10$ & $10-20$ & $0-10$ & $10-20$ & $0-10$ & $10-20$ & $0-10$ & $10-20$ \\
\hline CTC (cmolc dm-3) & 15,422 & 13,079 & 3,989 & 2,254 & 43,809 & 20,894 & 18,71 & 12,955 \\
\hline PST $(\%)$ & 20,450 & 19,160 & 16,720 & 22,160 & 39,010 & 39,400 & 43,78 & 44,670 \\
\hline $\mathrm{CE}\left(\mathrm{dS} \mathrm{m}^{-1}\right) 1: 1$ & 3,370 & 1,620 & 1,520 & 1,050 & 11,900 & 5,760 & 6,05 & 3,200 \\
\hline $\mathrm{pH}\left(\mathrm{H}_{2} \mathrm{O}\right) 1^{\prime}: 2,5$ & 7,130 & 7,559 & 6,590 & 6,230 & 7,200 & 7,500 & 7,97 & 8,280 \\
\hline $\mathrm{CO}\left(\mathrm{g} \mathrm{kg}^{-1}\right)$ & 19,846 & 10,046 & 7,805 & 3,358 & 19,846 & 10,046 & 9,91 & 5,049 \\
\hline Areia (\%) & 42,470 & 39,890 & 88,410 & 88,520 & 42,470 & 39,890 & 63,68 & 67,440 \\
\hline Silte (\%) & 37,460 & 38,680 & 5,700 & 4,010 & 37,460 & 38,680 & 22,39 & 18,300 \\
\hline Argila (\%) & 20,010 & 21,440 & 5,920 & 7,510 & 20,100 & 21,440 & 13,82 & 14,270 \\
\hline
\end{tabular}

CTC - Capacidade de troca de cátion; PST - Porcentagem de sódio trocável; CE - Condutividade elétrica; CO- Carbono orgânico

(1) variáveis relacionadas com a salinização do solo $(\mathrm{pH}, \mathrm{CE}$, PST e CTC) e o grupo (2) variáveis constituídas pelos metais pesados (Al, B, Hg, Mn, Mo, Si, Zr, Cr, Fe, V, Ni, Pb, Ti, Zn, As, $\mathrm{Se}, \mathrm{Cd}$, $\mathrm{Co}$ e $\mathrm{Cu})$ de acordo com os procedimentos descritos por Dunteman (1984).

Foi realizada análise multivariada empregando técnicas de análise fatorial por componentes principais $(\mathrm{AF})$ para $\mathrm{o}$ entendimento de como os atributos interagem, para isso, foram considerados os atributos CTC, PST, pH, CE, Al, B, Hg, Mn, Mo, $\mathrm{Si}, \mathrm{Zr}, \mathrm{Cr}, \mathrm{Fe}, \mathrm{V}, \mathrm{Ni}, \mathrm{Pb}, \mathrm{Ti}, \mathrm{Zn}, \mathrm{As}, \mathrm{Se}, \mathrm{Cd}$, Co e $\mathrm{Cu}$, nas camadas de 0-10 e 10-20 cm, cujos valores originais foram normalizados para média igual 0 e variância igual a 1 , a fim de compor as variáveis utilizadas na $\mathrm{AF}$.

À matriz de correlação dos atributos com os componentes considerar-se-á o nível de significância de 0,05 de probabilidade para, então, selecionar os atributos tidos como significativos que apresentam alta correlação com o componente principal em que se encontram. $\mathrm{Na} \mathrm{AF}$, os fatores com autovalores maiores que 1,0 foram extraídos por componentes principais, e os eixos fatoriais foram rotacionados pelo método Varimax. Estabeleceu-se para este estudo o valor de 0,65 para cargas fatoriais significativas.

Para a construção do banco de dados foram utilizados os softwares Microsoft Office Access ${ }^{\circledast}$ (2007), Microsoft Office Excel $^{\circledR}(2007)$ e as análises estatísticas no IBM PASW Statistics 18.0 (2010).

\section{Resultados e Discussão}

\section{Análise de correlação canônica}

Os teores de As e Hg não foram analisados pela análise de correlação canônica nem análise fatorial, devido não terem sido detectados nos solos dos quatro perímetros irrigados em estudo. De acordo com Kabata-Pendias \& Mukherjee (2007), geralmente os teores encontrados em solos contaminados, principalmente por atividades agropecuárias, são baixos. Porém, tomando-se uma perspectiva em longo prazo podem ser encontrados teores elevados provenientes de um acúmulo ao longo de anos de utilização desse compartimento ambiental, sendo este aumento intensificado em áreas próximas de complexos industriais, urbanos e, também, nas áreas rurais de agricultura altamente tecnificada.

As Tabelas 4 e 5 apresentam as correlações e pares canônicos entre as características que definem os solos afetados por sais e teores de metais pesados. Observa-se que para o primeiro par
Tabela 4. Correlações canônicas e pares canônicos entre as características dos conjuntos I $(\mathrm{pH}, \mathrm{CE}, \mathrm{PST}$ e CTC) e II (Cu, Ni, Fe, Zn, Mn, Cr, Zr, V e Al) referentes aos 80 pontos do estudo

\begin{tabular}{cccccc}
\hline \multirow{3}{*}{ Conjunto } & \multirow{4}{*}{ Variáveis } & \multicolumn{4}{c}{ Pares canônicos } \\
\cline { 3 - 6 } & & $\mathbf{4}$ & $\mathbf{2}$ & $\mathbf{3}$ & $\mathbf{4}$ \\
\cline { 3 - 6 } C1 & pH & 0,51 & $-0,30$ & $-0,54$ & $-0,60$ \\
& CE & 0,40 & 0,90 & $-0,04$ & $-0,16$ \\
& PST & 0,57 & 0,54 & $-0,55$ & 0,28 \\
& CTC & 0,64 & 0,72 & 0,23 & $-0,16$ \\
\hline \multirow{4}{*}{ C2 } & Cu & 0,21 & 0,09 & 0,33 & $-0,40$ \\
& Ni & 0,26 & 0,18 & 0,55 & $-0,63$ \\
& Fe & 0,72 & 0,26 & 0,37 & $-0,38$ \\
& Zn & 0,35 & 0,06 & 0,61 & $-0,43$ \\
& Mn & 0,68 & $-0,02$ & 0,51 & $-0,40$ \\
& Cr & 0,76 & $-0,08$ & 0,27 & $-0,46$ \\
& Zr & 0,14 & $-0,11$ & $-0,56$ & 0,40 \\
& V & 0,64 & 0,30 & 0,41 & $-0,47$ \\
& Al & 0,77 & $-0,03$ & 0,43 & $-0,29$ \\
\hline \multirow{4}{*}{} & R - Canônico & $0,72 * *$ & $0,46 n s$ & $0,41 \mathrm{~ns}$ & $0,34 \mathrm{~ns}$ \\
& Qui-quadrado & 91,13 & 38,87 & 22,05 & 8,88 \\
& GL & 36,00 & 24,00 & 14,00 & 6,00 \\
\hline
\end{tabular}

GL - Grau de liberdade; ns - não significativo respectivamente. (1) Correlações canônicas $\geq$ 0,5 foram consideradas significantes para fins de interpretação.

Tabela 5. Correlações canônicas e pares canônicos entre as características dos conjuntos I $(\mathrm{pH}, \mathrm{CE}, \mathrm{PST}$ e CTC) e II ( $\mathrm{Pb}, \mathrm{Cd}, \mathrm{Se}, \mathrm{Si}, \mathrm{B}, \mathrm{Mo}$, Ti e Co) referentes aos 80 pontos do estudo

\begin{tabular}{cccccc}
\hline \multirow{3}{*}{ Conjunto } & Variáveis & \multicolumn{4}{c}{ Pares canônicos } \\
\cline { 3 - 6 } & & $\mathbf{4}$ & $\mathbf{2}$ & $\mathbf{3}$ & $\mathbf{4}$ \\
\cline { 3 - 6 } Correlações Canônicas & \\
\cline { 3 - 6 } C1) & pH & 0,50 & $-0,72$ & 0,04 & 0,48 \\
& CE & 0,58 & 0,09 & $-0,75$ & $-0,31$ \\
& PST & 0,43 & $-0,51$ & $-0,38$ & $-0,64$ \\
& CTC & 0,81 & 0,24 & $-0,46$ & $-0,28$ \\
\hline \multirow{6}{*}{ C2 } & Pb & 0,75 & $-0,26$ & 0,18 & $-0,24$ \\
& Cd & 0,20 & $-0,06$ & 0,28 & $-0,58$ \\
& Se & 0,55 & 0,36 & 0,45 & 0,27 \\
& Si & 0,26 & 0,63 & $-0,14$ & $-0,16$ \\
& B & 0,90 & 0,33 & $-0,19$ & $-0,18$ \\
& Mo & $-0,04$ & $-0,18$ & $-0,14$ & $-0,65$ \\
& Ti & 0,67 & 0,22 & $-0,18$ & 0,06 \\
& Co & 0,77 & 0,18 & 0,12 & 0,01 \\
\hline \multirow{4}{*}{} & R - Canônico & $0,71 * *$ & $0,60^{\star *}$ & $0,52 \mathrm{~ns}$ & $0,31 \mathrm{~ns}$ \\
& Qui-quadrado & 112,96 & 62,63 & 29,93 & 7,48 \\
& GL & 32 & 21 & 12 & 5 \\
\hline
\end{tabular}

GL - Grau de liberdade; ns - não significativo respectivamente. (1) Correlações canônicas $\geq$ 0,5 foram consideradas significantes para fins de interpretação. 
as correlações canônicas foram altamente significativas (0,01 de probabilidade) pelo teste de qui-quadrado $(0,72)$ (Tabela 4$)$. O conjunto (I) explica o conjunto (II), no primeiro par canônico. Os atributos do solo CTC $(\mathrm{R}=0,64)$ e PST $(\mathrm{R}=0,57)$ foram as variáveis mais importantes no conjunto $\mathrm{I}$, que explicam os teores de metais pesados do conjunto II (Al, Cr, Fe, Mn, V). Percebese ainda que independentemente da predominância da fração mineral dos solos, solos arenosos e argilosos, estas relações se estabelecem tanto pelo material de origem, como pela afinidade química que estes metais possuem com a CTC e PST. Os outros pares (2, 3 e 4$)$ não tiveram importância $(\mathrm{R}<0,5)$.

A CTC foi a variável que mais influenciou, variando de acordo com a textura do solo. Este atributo está intimamente ligado às concentrações dos íons trocáveis presentes na solução do solo e aos sítios de troca nas interfaces coloidais do sistema. Uma elevada CTC proporciona uma maior retenção do metal no solo (van Raij, 1969). A matéria orgânica, embora represente em média 5\% dos componentes sólidos, é responsável por cerca de 30 a 65\% da CTC dos solos minerais, e mais de 50\% da CTC de solos arenosos e orgânicos (Matos, 1996). Corroborando com os resultados obtidos por Paye et al. (2012), no estabelecimentos de valores de referência de metais pesados no Estado de Pernambuco, verificaram que o teor de metais pesados, pode variar, principalmente, em função da textura e do CO dos solos nos horizontes superficiais. Por outro lado, o $\mathrm{pH}$ exerce forte influência na dinâmica dos íons metálicos catiônicos $\left(\mathrm{Cu}^{2+}, \mathrm{Zn}^{2+}\right.$, $\left.\mathrm{Ni}^{2+}, \mathrm{Mn}^{2+}, \mathrm{Fe}^{2+}, \mathrm{Cr}^{2+}, \mathrm{Co}^{2+}, \mathrm{Pb}^{2+} \mathrm{e} \mathrm{Cd}^{2+}\right)$, sendo estes mais móveis em condições de $\mathrm{pH}$ baixo, principalmente em solos com elevado grau de intemperização, onde os grupos funcionais de superfície dos componentes coloidais são, na sua maioria, dependentes de $\mathrm{pH}$, especialmente os oxi-hidróxidos de ferro e alumínio e formas mal cristalizadas de $\mathrm{Fe}$ e $\mathrm{Al}$ apresentam (Rieuwerts et al., 2006). Portanto, em condições de acidez aumenta o potencial de lixiviação das formas solúveis dos compostos metálicos, principalmente em áreas com predominância de textura arenosa, materiais com baixa capacidade de adsorção e pouco afetados por sais (Fernandez et al., 2007).

A Tabela 5 indica que houve correlações canônicas significativas $(\mathrm{R}=0,71 ; \mathrm{P} \leq 1 \%)$ e $(\mathrm{R}=0,60 ; \mathrm{P} \leq 1 \%)$, para $\mathrm{o}$ primeiro e segundo pares canônicos, respectivamente, pelo teste do qui-quadrado. Assim, os dois pares canônicos são de interesse do estudo. Pelos coeficientes do primeiro par canônico ficou evidenciada a relação entre caracteres que definem os solos afetados por sais (conjunto I) CTC $(\mathrm{R}=0,81)$ e $\mathrm{CE}(\mathrm{R}=$ $0,58)$, com metais $B(R=0,90)$, Co $(R=0,77), P b(R=0,75)$, $\mathrm{Ti}(\mathrm{R}=0,67)$ e Se $(\mathrm{R}=0,55)$ caracteres do (Conjunto II); são determinantes no incremento destes elementos no solos. $\mathrm{Na}$ medida em que atributos CTC e CE aumentam elevam os teores de $\mathrm{B}, \mathrm{Co}, \mathrm{Pb}, \mathrm{Se}$.

Pelos coeficientes do segundo par canônico (Tabela 5), verifica-se dependência significativa entre os dois grupos de variáveis analisadas e que suas relações são explicadas. Estabeleceu-se a relação entre o atributo do conjunto I pH ( $\mathrm{R}=-$ $0,72)$ com o mineral do conjunto II Si $(\mathrm{R}=0,63)$, sendo esta relação inversamente proporcional, fato explicado por Alcarde (1992) onde o mesmo afirma que os silicatos comportam-se de maneira similar aos carbonatos no solo e são capazes de elevar o pH, neutralizando o Al trocável e outros elementos tóxicos.

Especificamente o B $(0,90)$ foi o elemento que obteve a maior relação com os atributos relacionados com a salinização (CTC, CE e pH). A relação estabelecida entre a CTC e CE com $\mathrm{o} \mathrm{B}$, pode ser atribuída à influência da matéria orgânica sobre a CTC e a salinidade sobre o teor de M.O. Uma vez que em solos com altos teores de sais, sobretudo o sódio, elevam o $\mathrm{pH}$ do solo e por hidrólise diminuem o conteúdo de M.O podendo transferir-se para as camadas superficiais por capilaridade. No que concerne ao $\mathrm{pH}$, os teores de $\mathrm{B}$ aumentam gradualmente e chega a um máximo em $\mathrm{pH}$ variando de 7-9, dependendo do tipo de solo e minerais. À médida que o intervalo de $\mathrm{pH}$ diminui, cai sua adsorção gradualmente até chegar a um valor mínimo (De Bussetti et al., 1995).

Com relação ao $\mathrm{Pb}(\mathrm{R}=0,75)$ suas interações com os colóides dos solos são predominantemente específicas e menos dependentes das cargas superficiais, envolvendo a troca de metais com ligantes presentes na superfície dos coloides, que formam ligações covalentes e são a causa pelo qual alguns solos adsorverem metais acima da sua capacidade prevista com base na CTC (Pierangeli et al., 2001).

Já a explicação para interação do Ti com os atributos do conjunto I pode estar baseada no seu caráter anfótero. A fração argila e matéria orgânica humificada do solo apresentam tanto cargas positivas quanto negativas, normalmente, com predomínio das últimas. Nos minerais de argila silicatados do tipo 2:1 as cargas originam-se em maior parte por substituição isomórfica de íons na rede cristalina. Desta forma, estas cargas são permanentes, assim, os cátions podem ser adsorvidos eletrostaticamente, pelas reações de protonação e desprotonação, em qualquer valor de $\mathrm{pH}$ do solo (Ismail et al., 2009). Assim, valores elevados de CTC total nos solos, confere grande potencial na retenção de cátions, inclusive metais pesados, portanto maior capacidade de adsorção e retenção dos mesmos.

À vista disso, verificou-se comportamento diferente dos metais quando houve aumento da salinidade. Estabeleceramse relações entre a concentração de sais solúveis $(\mathrm{CE}=0,58)$ com os seguintes metais: $\mathrm{B}, \mathrm{Co}, \mathrm{Pb}$, Ti e $\mathrm{Se}$. Tal fato pode partir das seguintes fundamentações: capacidade de complexação de sais ânions com os metais pesados; e pela concorrência de sais cátions, derivados de cargas positivas, com os metais, também de cargas positivas, pelos sítios de sorção sobre a fase sólida (Paalman et al., 1994).

\section{Análise fatorial}

Para garantir que as variáveis possuíssem correlações mínimas suficientes para justificar o seu uso na matriz de dados na Análise Fatorial (AF), foi utilizada a análise de correlação de Pearson $(p<0,05)$ para as 21 variáveis estudadas, entre atributos químicos do solo ( $\mathrm{pH}, \mathrm{CE}$ e PST e CTC) e os teores de $\mathrm{Zr}, \mathrm{Mn}, \mathrm{Al}, \mathrm{Hg}, \mathrm{Ar}, \mathrm{Fe}, \mathrm{Cr}, \mathrm{Zn}, \mathrm{Pb}, \mathrm{Ni}, \mathrm{Cu}, \mathrm{Mo}, \mathrm{Co}, \mathrm{V}, \mathrm{Se}, \mathrm{B}$ e $\mathrm{Ti}$, nas amostras de solos dos quatro perímetros em conjunto.

Logo, a análise fatorial dos dados, com método de extração por componentes principais, permitiu extrair cinco (5) fatores 
Tabela 6. Eixos fatoriais para atributos do solo relacionados com a salinidade e teores de metais pesados para as amostras de solos dos quatro perímetros irrigados do CE com suas respectivas cargas fatoriais, autovalores, variância total e variância acumulada

\begin{tabular}{|c|c|c|c|c|c|}
\hline \multirow{3}{*}{ Variável } & \multicolumn{5}{|c|}{ Eixo fatorial ${ }^{(1)}$} \\
\hline & 1 & 2 & 3 & 4 & 5 \\
\hline & \multicolumn{5}{|c|}{ Cargas fatoriais ${ }^{(2)}$} \\
\hline $\mathrm{Cu}$ & 0,695018 & 0,046253 & 0,460725 & $-0,147174$ & $-0,142286$ \\
\hline $\mathrm{Ni}$ & 0,709172 & 0,041883 & 0,207200 & $-0,096895$ & 0,295195 \\
\hline $\mathrm{Fe}$ & 0,932954 & 0,235511 & $-0,039213$ & 0,045365 & 0,136550 \\
\hline $\mathrm{Zn}$ & 0,744784 & 0,001111 & $-0,016752$ & $-0,033545$ & 0,547683 \\
\hline $\mathrm{Mn}$ & 0,893521 & 0,086566 & 0,022458 & 0,107875 & 0,185061 \\
\hline $\mathrm{Cr}$ & 0,954252 & 0,102884 & $-0,069440$ & 0,164863 & 0,028820 \\
\hline $\mathrm{Zr}$ & $-0,082173$ & 0,042923 & $-0,569291$ & 0,124393 & $-0,678867$ \\
\hline V & 0,887386 & 0,219861 & 0,006855 & 0,042092 & 0,354608 \\
\hline $\mathrm{Al}$ & 0,955483 & 0,121763 & $-0,089806$ & 0,114553 & 0,100979 \\
\hline $\mathrm{Pb}$ & 0,771865 & 0,171876 & $-0,357657$ & 0,247060 & 0,058776 \\
\hline $\mathrm{Cd}$ & 0,466474 & $-0,022002$ & $-0,123723$ & 0,449368 & 0,627120 \\
\hline Se & 0,833002 & $-0,065295$ & 0,246359 & 0,047839 & 0,043747 \\
\hline $\mathrm{Si}$ & 0,396386 & 0,110001 & 0,189181 & $-0,220416$ & 0,804060 \\
\hline B & 0,597999 & 0,476725 & 0,016542 & $-0,012523$ & 0,346722 \\
\hline Mo & 0,109096 & 0,072426 & $-0,867156$ & $-0,135037$ & $-0,177771$ \\
\hline $\mathrm{Ti}$ & 0,778662 & 0,221787 & 0,091679 & $-0,002044$ & 0,484026 \\
\hline Co & 0,903153 & 0,157539 & 0,015765 & 0,061064 & 0,347593 \\
\hline $\mathrm{pH}$ & 0,196316 & 0,055227 & 0,083913 & 0,911576 & $-0,118955$ \\
\hline CE & 0,116027 & 0,959717 & 0,005720 & $-0,055514$ & 0,052209 \\
\hline PST & 0,040665 & 0,790084 & $-0,181394$ & 0,317473 & $-0,105081$ \\
\hline CTC & 0,286518 & 0,890057 & 0,011634 & $-0,032763$ & 0,086332 \\
\hline Autovalores & 11,037070 & 2,668920 & 1,981030 & 1,163450 & 1,060160 \\
\hline Variância total (\%) & 52,557470 & 12,709160 & 9,433480 & 5,540220 & 5,048360 \\
\hline Variância acumulada (\%) & 52,557470 & 65,266620 & 74,700110 & 80,240330 & 85,288690 \\
\hline
\end{tabular}

(1) Eixos fatoriais rotacionados pelo método Varimax. (2) Cargas fatoriais $\geq 0,65$ foram conconsideradas significantes para fins de interpretação.

com autovalor acima de (1), além de explicarem juntos $85 \%$ da variação dos dados (Tabela 6). Elementos como o $\mathrm{Cu}, \mathrm{Ni}$, $\mathrm{Fe}, \mathrm{Zn}, \mathrm{Mn}, \mathrm{Cr}, \mathrm{V}, \mathrm{Al}, \mathrm{Pb}$, Se, Ti e Co tiveram maior peso no fator 1. Já os atributos relacionados com a salinidade (CE, PST e CTC) tiveram peso maior no fator 2 , o Mo e $\mathrm{Zr}$, como baixa intensidade de relação, no fator 3 e somente o Co no fator $4 \mathrm{e}$ o Si e $\mathrm{Zr}$ de forma inversamente proporcional no fator 5. Vale salientar que nenhuma das variáveis relacionadas à salinidade do solo teve alta correlação com os teores de metais quando se analisaram os solos dos perímetros individualmente, o qual se confirmou quando se consideraram os solos dos perímetros em conjunto, indicando que não existe nenhuma interferência entre variáveis isoladas relacionadas com a salinidade e a presença de metais no solo. Fato explicado por Zanello et al. (2009), no estudo sobre a influência do chorume na concentração de metais pesados no solo, verificaram que os baixos teores de metais pesados no solo foram em consequência dos elevados teores de cátions trocáveis, como o $\mathrm{Na}^{+}, \mathrm{Ca}^{++}, \mathrm{Mg}^{++}$saturando as cargas negativas das argilas e da matéria orgânica, o que dificulta a adsorção dos metais pesados. Não obstante, foi verificado a inter-relação entre os metais, evidenciada, principalmente pelo fator 1, corroborando com as associações propostas por Goldschmidt (1958), onde o mesmo ressalta que vários elementos metálicos apresentam afinidades com o Fe, Mn, Ti e V, ou, ainda, afinidades geoquímicas entre os elementos. De igual modo, Lamy et al. (2006), estudando migração de metais pesados em Luvissolos irrigados com água de esgoto, observaram que houve interações entre metais $(\mathrm{Zn}, \mathrm{Pb}, \mathrm{Cu}$ e $\mathrm{Cd}$ ) no solos, o que favoreceu a migração destes para horizontes subsuperficiais dos perfis dos solos estudados. Já Fadigas et al. (2006), encontraram correlações para os metais entre: $\mathrm{Cu}$ e Mn; Cd e Co; Cd e Fe; Co e Mn; Co e Zn; Fe e Mn; Fe e Zn e Mn e Ni, sugerindo que os metais menos móveis nos solos são: $\mathrm{Cd}, \mathrm{Co}$, $\mathrm{Cu}, \mathrm{Ni}$ e $\mathrm{Zn}$ e que estes estão associados aos elementos Fe Mn.

Por outro lado, Kabata-Pendias \& Pendias (2001) observaram que o $\mathrm{pH}$ apresenta uma grande influência na adsorção de metais e, de modo geral, quanto maior o pH maior é a adsorção do tipo covalente no solo. Afirmando que o aumento do $\mathrm{pH}$ resulta no aumento da CTC pela desprotonação dos grupamentos ácidos da matéria orgânica e dos grupamentos $\mathrm{OH}$ das bordas dos argilominerais, na formação de hidróxidos, carbonatos e fosfatos insolúveis com os metais pesados e na complexação mais efetiva pela matéria orgânica.

\section{Conclusões}

1. Os atributos do solo relacionadas com a salinidade CTC, pH, PST e CE contribuíram para explicar os teores de metais pesados nos solos dos quatro perímetros irrigados, quando analisados em conjunto; todavia, não houve explicação quando analisados de forma individual. Os atributos relacionados com a salinidade seguiram a ordem de importância: CTC > $\mathrm{pH}>\mathrm{PST}>\mathrm{CE}$.

2. De forma geral, foram verificadas correlações entre os elementos metálicos analisados neste estudo com o Fe, Mn, Al, Ti e $\mathrm{V}$, ou, ainda, afinidades geoquímicas entre os elementos, nos solos dos quatro perímetros irrigados do Ceará. 


\section{Agradecimentos}

À FUNCAP, pela bolsa de estudos, e a UFC pela disponibilidade dos laboratórios, CNPq e INCT Sal, pelos recursos disponibilizados.

\section{Literatura Citada}

ADECE - Agencia de Desenvolvimento do Estado do Ceará (ADECE). Perímetros públicos do estado do Ceará. Fortaleza: ADECE, 2011.20p.

Alcarde, J. C. Corretivos da acidez dos solos: Características e interpretações técnicas. São Paulo: Associação Nacional para Difusão de Adubos, 1992. 9p.

EMBRAPA - Empresa Brasileira de Pesquisa Agropecuária. Manual de métodos de análises de solo. 2.ed. Rio de Janeiro. 1997. 212p,

De Bussetti, S. G., Ferreriro, E. A., Helmy, A. K. Sorption of boron by hydrous Al-oxide. Clays and Clay Minerals, v.43, p.58-62, 1995.

Dunteman, G. H. Introduction to multivariate analysis. London: Beverly Hills, 1984.

Fadigas, F. S.; Sobrinho, N. M. B. A.; Mazur, N.; Anjos, L. H. C.; Freixo, A. A. Proposição de valores de referência para a concentração natural de metais pesados em solos. Revista Brasileira de Engenharia Agrícola e Ambiental, v.10, p.699705, 2006.

Fadigas, F. S.; Sobrinho, N. M. B. A.; Mazur, N.; Anjos, L. H. C.; Freixo, A. A. Concentrações naturais de metais pesados em algumas classes de solos brasileiros. Bragantia, v.61, p.151-159, 2002.

Fernandez, R. O.; Cervera, J. V. G.; Vanderlinden, K.; Bojollo, R. C.; Fernández, P. G. Temporal and spatial monitoring of the $\mathrm{pH}$ and heavy metals in a soil polluted by mine spill. Post cleaning effects. Water Air Soil Pollution, v.178, p.229243, 2007.

Goldschmidt, V. M. Geochemistry. London: Oxford University Press, 1958. 425p.

Ismail, A. A.; Bahnemann, D. W.; Bannat, I. M. Wark Gold nanoparticles on mesoporous interparticle networks of titanium dioxide nanocrystals for enhanced photonic efficiencies. Journal of Physical Chemistry, v.113. p.74297435, 2009.

Kabata-Pendias, A.; Mukherjee, A. B. Trace elements form soil to human. New York: Springer. 2007. 550p.
Kabata-Pendias, A.; Pendias, H. Trace elements in soils and plants. 3.ed. Boca Raton, Florida: CRC Press. 2001.315p.

Lamy, I.; Oort, F.; Deré, C.; Baize, D. Use of major and traceelement correlations to assess metal migration in sandy Luvisols irrigated with wastewater. European Journal of Soil Science, v.57, p.731-740, 2006.

Leite, E. M.; Cavalcante, L. F.; Diniz, A. A.; Santos, R. V.; Alves, G. S.; Cavalcante, I. H. L. Correção da sodicidade de dois solos irrigados em resposta à aplicação de gesso agrícola. Irriga, v.12, p.168-176, 2007.

Matos, A. T.; Fontes, M. P. F.; Jordão, C. P.; Costa, L. M. R. Mobilidade e formas de retenção de metais pesados em latossolo vermelho-amarelo. Revista Brasileira de Ciência do Solo, v.20, p.379-386, 1996.

Paalman, M. A. A., van der Weijden, C. H.; Loch, J. P. G. Sorption of cadmium on suspended matter under estuarine conditions: competition and complexation with major seawater ions. Water Air Soil Pollution, v.73, p.49-60, 1994.

Payê, H. S.; Vargas, J. W.; Mello, S. B. Métodos de análise multivariada no estabelecimento de valores de referência de qualidade para elementos-traço em solos. Revista Brasileira de Ciência do Solo, v.36, p.1031-1041, 2012

Pierangeli, M. A. P.; Guilherme, L. R. G.; Curi, N.; Silva, M. L. N.; Oliveira, L. R.; Lima, J. M. Teor total e capacidade máxima de adsorção de chumbo em Latossolos brasileiros. Revista Brasileira de Ciência do Solo, v.25, p.279-288, 2001.

Rieuwerts, J. S.; Ashnore, M. R.; Farago, M. E.; Thornton, I. The influence of soil characteristics on the extractability of $\mathrm{Cd}$, $\mathrm{Pb}$ and $\mathrm{Zn}$ in upland and moorland soils. Science of the total Environment, v.366, p.864-875, 2006.

USEPA - US Environmental Protection Agency. Method 3050 B. 1998. <http://www.epa.gov/SW-846/pdfs/3050b.pdf>. 10 Jan. 2014.

van Raij, B. A capacidade de troca de catíons das frações orgânica e mineral em solos. Bragantia, v.28, p.85-112, 1969.

Zanello, S.; Melo, V. F. Mineralogia e teores de cromo, níquel, cobre, zinco e chumbo nos solos no entorno do aterro sanitário da Caximba em Curitiba-PR. Scientia Agraria, v.10, p.51-60, 2009.

Yongming, H.; Peixuan, D.; Junji, C.; Posmentier, E. S. Multivariate analysis of heavy metal contamination in urban dusts of XI'an, Central China. Science Total Environment, v.355, p.176-186, 2005. 